\title{
Le devenir de la famille paysanne de la réforme agraire dans le Saïss au Maroc sous une perspective de genre
}

\author{
Lisa Bossenbroek \\ Faculté de Gouvernance et des Sciences Economiques et Sociales \\ (CRESC, EGE RABAT), Morocco \\ lisabossenbroek@gmail.com
}

\begin{abstract}
In 2006, the government of Morocco decided to privatize the land of the former socialist-inspired collective state cooperatives, which had been created in the early 1970 s. The resulting shift in land control is happening alongside - but is also provoking wider processes of agrarian change, manifested among others things in land sells, the introduction of new high-value crops, the use of new technologies (tubewells and drip irrigation), and alterations in labour relations. These transformations influence the development of family farming and gender relations. On the basis of an ethnographic and historical study conducted in a dissolving state cooperative in the agricultural plain of the Saïss we suggest opening the "black box" of the family farm to illustrate the gender dynamics that mark the impact of current agrarian dynamics today. Through this lens we first of all illustrate how during the period of the state cooperative the land, the farms, and the families were deeply intertwined through labour relations and by how the land was used, forming the identities of peasant women and men. By retracing the development of various peasant families we illustrate how this farming model is changing today. The current agrarian dynamics offer new possibilities of being and becoming to some young men and "modern" farmers. Nevertheless, various
\end{abstract}

* L'élaboration de cet article a bénéficié de l'aide précieuse de Zakaria Kadiri, Mohamed Mahdi, Larbi Zagdouni, Ahmed Bendella et Anne Bossenbroek-Bouchard qui ont pris le temps de relire la première version de ce travail et m'ont fait part de leurs remarques et suggestions. Je tiens aussi à remercier énormément les différentes familles avec qui je me suis entretenue. Sans leur disponibilité et aide, ce travail n'aurait pas pu aboutir.

** Faculté de Gouvernance et des Sciences Economiques et Sociales (EGE Rabat), Centre de Recherche Economie Société et Culture (CRESC), Maroc. 
peasant women increasingly find less pride in their agricultural work and actively seek to develop new rural feminine identities, which is not easy. As such, we observe how the future becoming of peasant farming will strongly depend on the next generation of farmers and of their aspiration of modernizing their future becoming.

\section{Keywords}

agrarian change - land dynamics - peasant farming - gender - rural youth Morocco

\section{Résumé}

L'État marocain décida en 2006 de privatiser les terres des coopératives de la réforme agraire créée au début des années 1970. Cette dynamique foncière s'inscrit dans des processus plus larges de changements agraires, qui se manifeste entre autre par la vente des terres, l'introduction de nouvelles cultures à forte valeur ajoutée, l'utilisation de nouvelles technologies (forage et goutte-à-goutte) et des transformations dans les relations de travail. Ces changements auront une influence sur le développement de l'agriculture familiale et les relations de genres. À la base d'un travail ethnographique et historique portant sur une coopérative dans la plaine du Saïss, nous suggérons d'ouvrir la «boite noire» de l'agriculture familiale pour illustrer les dynamiques de genre qui marquent aujourd'hui l'impact des changements agraires. À travers ce regard nous illustrons dans un premier temps comment, pendant la période de la coopérative la terre, l'exploitation et la famille paysanne étaient intimement liées. Les activités et l'organisation autour de la terre forment les identités des hommes et des femmes paysans. Aujourd'hui, nous illustrons au travers de différentes trajectoires de familles paysannes comment ce modèle est en mutation. Les dynamiques agraires offrent de nouvelles opportunités à certains jeunes hommes et agriculteurs «modernes». Cependant, différentes femmes paysannes ne trouvent plus de fierté dans le travail agricole et essaient de développer de nouvelles identités féminines rurales, ce qui n'est pas facile. Ainsi, nous verrons que le devenir de l'agriculture paysanne dépendra fortement de cette nouvelle génération de jeunes agriculteurs et agricultrices et de leurs aspirations à moderniser leur devenir.

\section{Mots clés}

changements agraires - dynamiques foncières - agriculture paysanne - genre jeunesse rurale - Maroc 


\section{Introduction}

Dans un monde globalisé dans lequel les sociétés rurales et agricoles sont mêlées dans des dynamiques internationales, le devenir de l'agriculture familiale est de nouveau mis en discussion. Dans ce débat, les relations complexes au sein de l'agriculture familiale sont souvent simplifiées en réduisant la famille paysanne à un acteur, en général, le chef de famille (masculin) qui agit au nom de l'intérêt de tous les membres de la famille ${ }^{1}$. Ainsi, la femme paysanne n'est pas prise en compte, ni les rapports de genre et les relations générationnelles.

Nous proposons d'ouvrir à nouveau la «boite noire» concernant l'exploitation familiale en considérant l'agriculture familiale comme une juxtaposition complexe de relations de famille, de parenté, marquée par les rapports de genre, de pouvoir et de générations, fondée autour de la terre ${ }^{2}$. Sous cet angle, à travers différents travaux portant sur le genre et l'exploitation familiale, nous analyserons l'implication de la femme rurale au sein de l'exploitation. Ces travaux montrent comment les tâches agricoles et domestiques sont complémentaires et divisées selon le genre, lâge et la place de chacun dans la parenté, illustrant l'association de la famille et l'exploitation ${ }^{3}$. Ils mettent en lumière aussi le fait que dans le contexte de la modernisation du domaine agricole, les relations de genre et les identités des hommes et des femmes mutent ${ }^{4}$. Dans un contexte de modernisation de l'agriculture, ils mettent en valeur, par exemple, comment le travail masculin est de plus en plus défini par des compétences de gestion et d'entreprenariat permettant à l'agriculteur de se transformer en « entrepreneur » alors que certaines femmes cherchent à professionnaliser leur travail et, par là, leur identité par le biais de se déclarer « agricultrice à titre principal » et non plus « aide familiale $»^{5}$.

Si cette littérature se prête aux analyses de genre de telles dynamiques agraires au niveau international, les recherches sur les mêmes sujets au Maghreb sont peu nombreuses ${ }^{6}$. Toutefois, plusieurs travaux qui portent sur le Maroc montrent comment l'impact de la modernisation sur les mutations sociales, culturelles et économiques réorganise les rapports et comment les identités de genre contribuent à la recomposition des rapports familiaux ${ }^{7}$.

\footnotetext{
1 Whatmore 1991; Argent 1999.

2 Whatmore 1991.

3 Whatmore 1991; Zwarteveen and Endeveld 1995.

4 Asztalos et Brandth 2007 ; Brandth 2002 ; Ní Laoire 2002.

5 Barthez 2005.

6 Mernissi 1982; Mernissi 1983; Belarbi 1995; Bossenbroek, Van der Ploeg, and Zwarteveen 2015 .

7 Anglade, Bouasria, Cheikh, Debarre, Manry et Schmoll 2013.
} 
Ainsi, la réorganisation de la division sexuelle du travail et les changements des rapports sociaux laissent apparaître des manières inédites de se positionner pour différents femmes et hommes et de renégocier leur identité et leurs destins sociaux ${ }^{8}$.

En nous appuyant sur cette littérature, nous chercherons à comprendre, à travers les dynamiques agraires actuelles, comment les relations de genre et les identités des hommes et des femmes se transforment et se réarticulent. Plus particulièrement, nous analyserons sous cette perspective le devenir d'une agriculture familiale d'un genre particulier et qui a émergé dans un contexte historique singulier, à savoir dans le cadre de la réforme agraire. Initiée au début des années 1970, cette réforme correspond à la politique de redistribution des terres récupérées des anciens colons acquises au temps du protectorat Français (1912-1956). L'objectif de ladite réforme était la mise en place d'une nouvelle classe de paysans en leur attribuant des exploitations viables sur lesquelles les familles bénéficiaires seraient capables de participer activement au développement agricole envisagé par l'État. Les adhérents membres de ces coopératives étaient soumis à des règles strictes de gestion collective tout en restant sous le contrôle de l'État. En 2006, l'État marocain choisit de libéraliser le secteur et de le «melkiser $»^{9}$ en donnant aux adhérents la possibilité d'obtenir leur titre foncier privé dans l'objectif de lever les interdictions et les obligations contraignantes qui entravaient la mise en valeur des terres ${ }^{10}$. Aujourd'hui, le secteur s'inscrit dans un modèle libéral de développement et une partie de ces terres est actuellement mise sur le marché. Ce modèle donne naissance à une agriculture entrepreneuriale aux dépens de l'agriculture familiale ${ }^{11}$. C'est dans ce contexte que nous analysons le devenir d'une agriculture familiale tout en se demandant ce que signifient les changements actuels pour les différents acteurs ruraux qui sont aujourd'hui concernés par ces dynamiques.

À travers une analyse historique d'une coopérative établie lors de la réforme agraire dans la plaine du Saïss au Maroc, nous étudions dans un premier temps comment, pendant la période de la coopérative, la terre était exploitée par des

8 Bouasria et Cheikh 2013 .

9 «Melkiser» fait référence au changement du statut foncier de la terre. D'une terre de la coopérative de la réforme agraire où les adhérents avaient uniquement le droit d'usage de la terre, les adhérents vont, en répondant à différents critères, avoir l'opportunité d'obtenir le titre foncier de la terre qu'ils exploitaient. Ainsi, la terre devient une terre «melk», une propriété privée.

10 Les décrets avec les modifications du statut de ces terres ont été publiés dans les bulletins officiels $n^{\circ} 5282$ du 13 janvier 2005 et $n^{\circ} 5446$ du 10 août 2006.

11 Bossenbroek and Zwarteveen 2015. 
familles paysannes et par la communauté dans une structure familiale renforcée par les mariages et le système d'héritage. Par la suite, nous mettons en lumière comment après la libéralisation des terres des coopératives de la réforme agraire, ces liens s'érodent graduellement et reconfigurent les relations de genre amenant un impact sur les identités des hommes et des femmes du milieu paysan. Nous illustrons ceci à travers la présentation de trois typologies de devenir de familles paysannes : 1) celles forcées de vendre leurs terres ; 2) celles cherchant à se «moderniser»; 3) celles restant sur leurs terres et poursuivant une agriculture « traditionnelle » sans plan d'avenir.

\section{Méthode}

Cette recherche s'appuie sur une étude ethnographique ${ }^{12}$ effectuée entre 2012 et 2015 au sujet d'une coopérative établie en 1972 à l'époque de la réforme agraire. Nommée Ait Ali, cette coopérative se trouve dans la plaine du Saïss. Les terres ayant servi à la création de cette coopérative, d'une superficie totale d'environ $35^{\circ}$ hectares, ont été réparties entre 36 attributaires, dont 3 femmes, qui y habitent avec leur famille. La majorité des attributaires sont originaires du douar ${ }^{13}$ d'Ait Ali, à l'exception de quelques adhérents qui viennent d'autres villages situés dans la plaine du Saïss. La coopérative fait partie du douar d'Ait Ali, elle est composée d'environ 80 familles élargies. La majorité des familles sont Amazighs et appartiennent à la tribu Ait Naamen.

Le premier objectif de notre étude était de retracer les trajectoires des différentes familles paysannes d'un point de vue historique. Nous avons pu mettre en lumière trois situations grâce aux expériences des différents membres des familles habitant dans la coopérative. Nous avons aussi tenu compte du rapport à la terre et de l'organisation de la main d'œuvre sur l'exploitation. Notre deuxième objectif était d'analyser les pratiques autour de la libéralisation du secteur de la réforme agraire et, grâce aux expériences vécues par les personnes concernées, de nous interroger sur l'émergence de nouvelles inégalités liées à la répartition de la terre et aux pratiques agricoles.

Pour ce faire, nous avons utilisé différentes techniques d'entretien: écoute des histoires de vie, entretiens abordant un sujet précis (une opinion

12 Bossenbroek 2016.

13 Le douar est officiellement défini comme étant: «Un ensemble de foyers réunis par des liens réels ou fictifs de parenté, correspondant à une cellule territoriale, comportant ou non des modes d'exploitation communautaires et dirigé autant que possible par un moqaddem » (Circulaire du Ministère de l'Intérieur en date du 27 décembre 1964). 
personnelle par rapport à un événement, à un changement dans la société ou encore à une projection dans l'avenir) et des groupes de discussions. Beaucoup de personnes se montraient réticentes au fait de partager avec nous leurs histoires intimes concernant leur terre. C'est pourquoi nous avons consacré plus de temps à des visites personnelles et nous avons pris part à des événements sociaux importants, tels que les mariages, les rites de naissance, l'Eid el Sghir ${ }^{14}$ ainsi que l'Eid el Kebir ${ }^{15}$, essayant de nous faire accepter dans leur communauté. Grâce à cela, nous avons découvert que la vente des terres peut être un acte très douloureux, considéré comme honteux et générant beaucoup de tristesse. C'est en partie pour cette raison que le recueil des témoignages demande beaucoup de patience, d'écoute et de finesse.

\section{Le contexte de la plaine du Saïss}

La plaine du Saïss, située entre les villes de Meknès et Fès, couvre une superficie de 220000 hectares dont 49677 sont irrigués (Ministère de l'Agriculture 2012). Au Saïss, 93 coopératives furent créées entre 1969 et 1977, représentant environ $12 \%$ des coopératives créées à l'échelle nationale ${ }^{16}$.

L'agriculture est l'activité principale de la plaine. Dès les années 1980, elle a connu une dynamique encouragée par les politiques de libéralisation, par l'accès à l'eau souterraine grâce au creusement de puits et de forages dont le nombre s'est accru rapidement (ce qui a permis le remplacement des cultures pluviales par des cultures irriguées), par l'ouverture et l'accès à de nouveaux marchés et par l'usage de nouvelles technologies (goutte-à-goutte, forage). Depuis 2008, le secteur agricole bénéficie d'une nouvelle impulsion grâce au «Plan Maroc Vert», un plan stratégique qui a pour ambition la modernisation rapide et l'intensification de l'agriculture marocaine. Une première appréciation montre que ce plan favorise l'agriculture moderne à haute valeur ajoutée et de haute productivité, l'adoption de nouvelles technologies et l'intensification de la production ${ }^{17}$.

\footnotetext{
14 La fête qui indique la fin du Ramadan.

15 La fête du sacrifice.

16 Allali et Mahdi 2001.

17 Akesbi 2012.
} 


\section{Les coopératives de la réforme agraire : l'histoire d'une évolution}

Le débat autour de la réforme avait débuté après l'indépendance du Maroc en 1956 et portait sur le devenir des terres colonisées, équivalentes à un million d'hectares. Dès les premières années de l'indépendance, l'État procéda à des distributions modestes. Les premières expériences de réforme agraire ont été faites sans doctrine générale et avant que ne soient publiés les grands textes du 4 juillet 1966 sur la réforme agraire et du 25 juillet 1969 sur le Code des Investissements Agricoles ${ }^{18}$.

Le grand lot de terres récupérées par l'État restait sujet à des débats politiques. Les partis de gauche étaient persuadés qu'une vraie réforme agraire s'avérait indispensable, militant pour le démantèlement des grandes fermes et domaines coloniaux et leur redistribution aux petits agriculteurs et paysans sans terre ${ }^{19}$. Cependant, les pouvoirs publics étaient réticents à de grandes réformes qui changeraient les enjeux des pouvoirs locaux. Il s'en est suivi une redistribution des terres au début des années 1970 mais qui n'a concerné que $30 \%$ du million d'hectares récupéré ${ }^{20}$. Cette réforme agraire a été dirigiste et étroitement contrôlée par l'État ${ }^{21}$ à travers le regroupement d'ouvriers et de petits paysans dans les coopératives étatiques, dites «les coopératives de la réforme agraire », placées sous la tutelle du Ministère de l'Intérieur. En théorie, les femmes pouvaient, elles aussi, devenir adhérentes mais en pratique, nos entretiens révèlent que lorsqu'elles s'inscrivaient, elles étaient uniquement sélectionnées lorsque leur mari n'était pas éligible ${ }^{22}$.

L'objectif de cette politique était la mise en place d'une nouvelle classe de paysans et la formation d'exploitations agricoles familiales viables, capables de participer activement au développement agricole. La terre distribuée restait propriété de l'État. Plus spécifiquement, il s'agit d'une vente de terre sous condition particulière (Dahir de la Réforme Agraire 29 décembre 1972 article 9). Ainsi, l'attributaire ne devenait pleinement propriétaire qu'après s'être acquitté du remboursement de la totalité de son lot et avoir satisfait ses obligations. Le paiement du prix des lots était échelonné sur 20 ans avec remboursement par annuité au taux d'intérêt de $4 \%$ par an. Néanmoins, très peu d'attributaires

\footnotetext{
18 Bouderbala et Filali-Meknassi 1991, p. 86.

19 Bouami 1980.

20 Bouderbala 2001.

21 Bouderbala et Filali-Meknassi 1991.

22 Par exemple, lorsque le mari avait émigré en France, il n'était pas éligible pour devenir adhérent. Les règles d'adhésion étaient établies dans le Dahir de la Réforme Agraire qui date du 29 décembre 1972.
} 
ont réussi à obtenir leur lot au bout de 20 ans et ce, pour des raisons diverses : 1) les termes du prix n'étaient pas acquittés ; 2) l'attributaire n'avait pas satisfait aux clauses du cahier de charges ; 3) l'acte de vente n'avait pas pu être établi du fait, principalement de la difficulté à fixer le prix du lot lorsqu'il faut en déduire la valeur des terres que l'attributaire possédait avant l'attribution et qu'il devait céder à l'Etat ${ }^{23}$. L'adhérent était d'autant plus soumis à des règles strictes. Ainsi, il avait l'obligation de travailler son lot en famille et d'adhérer à la coopérative. L'absentéisme, le mode de faire-valoir indirect et même l'utilisation de salariés permanents étaient interdits ${ }^{24}$. Les lots étaient impartageables, incessibles, insaisissables et inaliénables sauf au profit de l'État. Pour éviter tout morcellement, en cas de décès de l'attributaire, le lot était attribué à un seul héritier, qui, lorsqu'il/elle décidait de pratiquer l'agriculture tout seul et de ne plus exploiter la terre avec le reste de la famille au bénéfice de tous, était obligé d'indemniser ses cohéritiers ${ }^{25}$.

Dans la plaine du Saïss, trois différents types de coopératives ont été créées. Dans les coopératives mixtes et de services, les adhérents recevaient un lot individuel alors que dans les coopératives de production, qui faisaient environ 3 ooo hectares, la terre était maintenue en copropriété et les adhérents travaillaient comme ouvriers, rémunérés annuellement. Dans le Saïss, trois coopératives de production ont été créées. Au début des années 1990, ces coopératives ont été divisées en plusieurs petites coopératives et sont devenues des coopératives de services et des coopératives mixtes. Ces coopératives étaient censées faciliter l'accès aux facteurs de production nécessaires à la mise en valeur des lots et à la commercialisation de la production.

En bref, cette réforme agraire a été un acte isolé, sans remise en question des structures du pouvoir et des modes de production ${ }^{26}$. Les adhérents des coopératives nétaient pas libres d'entrer et de sortir de ces coopératives. Ils étaient soumis à des règles strictes, obligations et contraintes et devenaient des clients obligés de l'administration ${ }^{27}$.

23 Entretien avec un responsable de la Directions Provinciales de l'Agriculture (DPA) à El Hajeb 30 avril 2012 ; voir aussi Bouderbala et Filali-Meknassi 1991, p. 88.

24 Bouderbala et Filali-Meknassi 1991.

25 Idem.

26 Pascon 1977.

27 Idem. 


\section{La période de la coopérative : émergence d'une symbiose entre la terre et la famille ${ }^{28}$}

La coopérative d'Ait Ali est née de l'éclatement d'une grande coopérative de production. À sa création, chaque adhèrent reçut un lot individuel de 7 à 13 hectares en fonction de la qualité de la terre dont un lot de 0,5 hectares irrigué, où cultiver les légumes et un lot pour l'habitat. Une analyse des trajectoires historiques des différentes familles paysannes vivant dans cette coopérative ainsi que de leurs pratiques montrent comment les adhérents se sont positionnés dès la réception de leur lot individuel. Ainsi, comme nous le verrons dans la section suivante, une symbiose se réalise entre la terre et les familles qui la travaillaient. Cet ancrage de la famille à la terre se développe au travers du système d'héritage particulier aux coopératives, le mariage et l'organisation du travail entre familles. Cela régit les relations de genre ainsi que les rapports sociaux autour de la terre.

\section{Un système d'héritage particulier}

Lors du décès de l'attributaire, l'adhésion à la coopérative et le droit d'usage de la terre revenait à un héritier pour éviter le morcellement de la terre et pour garder la continuité de l'exploitation dans la famille. Lorsque le titre du lot était inscrit au nom du chef de la famille (masculin), le titre revenait à sa femme. Nos interlocuteurs justifiaient cela par le rôle particulier de la mère dans la famille et dans la société (rurale) marocaine. La mère est considérée comme l'épine dorsale de la famille, elle est très honorée et vénérée et elle est perçue comme forte et responsable ${ }^{29}$. Inscrire la terre au nom de la mère était une stratégie pour maintenir la cohésion au sein de la famille et éviter les conflits entre frères et sœurs. C'était aussi une mesure ayant pour but d'empêcher le morcellement de la terre: "La femme, c'est elle qui coiffe tout. Si un garçon prend le lot, il chassera sa mère et ses frères, s'il se marie, sa femme ne voudra plus vivre avec eux. C'est pourquoi l'État a donc pensé à attribuer le lot à la mère parce que celle-ci comme le père auparavant est responsable de tout ${ }^{30}$. C'est ainsi que dans la coopérative d'Ait Ali, le nombre d'adhérents féminins a augmenté au fil des années et atteint aujourd'hui neuf sur trente-six.

28 Quelques aspects dans ce paragraphe ont été abordés dans un chapitre de l'ouvrage: Bossenbroek and Zwarteveen 2015.

29 Rassam 1980.

30 Entretien avec un ancien directeur d'une coopérative de la réforme agraire effectué le 7 mai 2012 dans la commune rurale de Bettit (dans la plaine du Saïss), en français par l'auteur. 
Lorsqu'une mère de famille ne pouvait hériter de la terre, cette dernière était souvent transférée à un fils non marié vivant encore sous le toit de ses parents. Cette stratégie était adoptée afin de maintenir la terre dans la famille : si la terre était transférée à un fils marié, la famille risquait de la perdre lors du décès de celui-ci car, alors, elle reviendrait à sa femme. Nos entretiens montrent que la terre, dans ce cas aussi, continuait à être exploitée par toute la famille et l'indemnisation aux autres héritiers n'était que rarement effectuée. De ce fait, les règles et pratiques adoptées, dans le cadre de la réforme agraire, contribuaient ainsi à consolider la famille et à renforcer sa reproduction sociale et son ancrage à la terre.

\section{Le mariage et les arrangements de travail}

L'institution du mariage contribuait à la désignation des identités ainsi qu'à la régulation des rapports de travail et de genre autour de la terre. Au moment du mariage, la jeune épouse quitte généralement la maison et la terre parentale et s'installe chez sa belle-famille (Encadré ${ }^{\circ}{ }^{1}$ ).

\section{Encadré $\mathrm{n}^{\circ}$ : : Aziza, l'épouse qui se forge une identité}

Le cas d'Aziza ${ }^{31}, 5^{0}$ ans, illustre bien ce propos. À 16 ans, Aziza a épousé son mari Hassan. Le plus jeune frère de Hassan est adhérent de la coopérative. La terre se trouve à un kilomètre de leur maison. Aziza est originaire d'une terre appartenant à une autre coopérative de la réforme agraire où elle est née et a grandi jusqu'à son mariage ; cette terre est située à environ 40 kilomètres d'Ait Ali. Elle essaie de maintenir son droit d'accès à sa terre natale en envoyant son fils travailler sur celle-ci. Elle nous expliquait qu'elle faisait ainsi pour pouvoir s'assurer de la transmission de la part de la terre qui lui reviendrait après le décès de ses parents pour ainsi pouvoir la transmettre plus tard à ses enfants ${ }^{32}$.

Comme toutes les autres familles lors des premières années de la création de la coopérative, la famille d'Aziza, au sein de la coopérative de son mari, cultivait des céréales, du blé, de l'orge et des pois. Le semis

Tous les noms propres utilisés dans cet article ont été changés pour garder l'anonymat des personnes interviewées. Les entretiens ont été effectués le 21 juin 2012, le 4 juillet 2012, le 28 février 2013 et le 4 novembre 2013 en langue darija par l'auteur avec l'aide d'une assistante de recherche/interprète marocaine. 
et la récolte étaient considérés comme un travail d'homme, effectué par son mari et ses beaux-frères, parfois aidés d'Aziza et de ses belles-sœurs. Aziza et les autres membres féminins de la famille étaient responsables de toutes les activités ménagères consistant en la préparation du petit déjeuner et des repas de la journée, en la préparation du pain ainsi que la lessive et le ménage. Elles devaient aussi s'occuper de leurs trois vaches. Elles étaient également responsables de trier le blé, l'orge et les pois chiches après la récolte.

En 2001, Hassan et ses frères ont investi dans le creusement d'un puits et ont commencé à faire des cultures irriguées sur trois hectares (oignon, pomme de terre, carotte, tomate). À la suite de ces changements, Aziza a commencé, de plus en plus, à apporter son aide dans le travail sur l'exploitation, parfois accompagnée de ses belles-sœurs ou d'autres membres de la famille élargie. Elle travaillait en compagnie de son mari, de ses beaux-frères et de leurs fils mais selon des tâches bien réparties selon le genre. La préparation de la terre et l'irrigation ainsi que la gestion de l'exploitation étaient considérées comme des activités masculines alors que les femmes se chargeaient de la plantation et du désherbage, tâches considérées comme des travaux féminins. La récolte était faite ensemble.

Un autre exemple (Encadré ${ }^{\circ}{ }^{2}$ ), celui de Molud, montre comment les identités sont, entre autres, exprimées et affirmées à travers la position qu'occupe une personne au sein de la famille ainsi que par la distribution des travaux agricoles selon le genre et lâge.

\section{Encadré $\mathrm{n}^{\circ} 2$ : Molud, le chef de famille}

Molud $^{33}, 65$ ans, reçut un lot de 8,5 hectares. Il a deux fils et une fille ayant aujourd'hui entre 25 et 30 ans. À l'origine, il pratiquait des cultures en bour (pluvial). Malgré le fait que c'était formellement interdit de faire appel à des ouvriers à l'extérieur de la coopérative, Molud, comme beaucoup d'autres familles appartenant à la coopérative, embauchait des ouvriers salariés du douar d'Ait Ali. Quelques années plus tard, il a creusé un puits et partit habiter avec sa famille sur son lot de la coopérative. Dès qu'ils eurent déménagé, ses deux fils ont arrêté leurs études et ont commencé

33 Les entretiens avec Molud ont été effectués le 9 octobre 2013, 10 novembre 2013 et 3 mars 2014 en darija par l'auteur avec l'aide d'une assistante de recherche/interprète marocaine dans la coopérative d'Ait Ali. 
à participer activement aux travaux sur l'exploitation. Ils plantèrent alors trois hectares d'oignons et un hectare et demi de tomates; le reste du terrain était utilisé pour cultiver du blé. Les tâches étaient ainsi réparties : le fils ainé était chargé de l'irrigation, de rassembler les ouvriers et de la gestion de l'exploitation tandis que le cadet s'était chargé de la conduite du tracteur (acheté par Molud) sur leur terre ainsi que sur les lots des voisins pour se faire un peu d'argent. Les deux frères nous ont confié «qu'ils prenaient toujours l'avis de leur père et discutaient de tout avec lui». Leur maman, amoindrie par des problèmes de santé, n'apportait pas d'aide sur l'exploitation. Leur sœur vivait avec une tante en ville où elle allait à l'école. Le travail domestique était effectué par une des belles-filles qui s'occupait aussi des quelques vaches qui leur appartenaient.

Ces deux exemples illustrent bien comment l'utilisation de la terre est ancrée dans les relations de famille, de parenté et de communauté au service de la reproduction du modèle de l'exploitation familiale. Cela donne forme et sens aux identités des paysans, hommes et femmes. Aziza estimait important de garder les liens avec sa terre natale : elle les considérait comme une manière de sécuriser le futur de ses enfants. Par ailleurs, entretenir l'accès et l'usage de la terre contribuait aussi à son autonomie personnelle. Parallèlement, ses activités sur la terre de son beau-frère étaient suffisamment importantes pour affirmer sa position sociale dans la famille de son mari; grâce à son travail, elle se procure une réputation et une identité de bonne épouse et de bonne mère. Le travail sur la ferme est construit et perçu comme une extension du travail domestique. Étant réalisé avec les membres de la famille, il permettait à des femmes comme Aziza d'étendre les limites de leur espace privé dans le monde rural du Maroc, culturellement et traditionnellement attribué à la femme. Cela lui permet d'élargir sa liberté sans toutefois perdre sa crédibilité en tant que femme, mère et/ou épouse. Pour les hommes, comme le montre le cas de Molud, les références identitaires sont beaucoup plus liées à la propriété foncière, au statut d'agriculteur et aux activités agricoles: l'homme est perçu comme le chef du foyer ou de l'exploitation alors que les références identitaires des femmes sont beaucoup plus liées au contrat de mariage et associées à la sphère privée de la maison ${ }^{34}$. 


\section{Dysfonctionnement des coopératives et libéralisation du secteur}

Les trajectoires historiques des différentes familles paysannes révèlent qu'au fil des années il se met en place une différentiation graduelle entre les différentes familles de la coopérative ${ }^{35}$. Certaines avaient les moyens de creuser des puits, transformant ainsi leur système de production, alors que d'autres s'endettaient ${ }^{36}$ pour pouvoir accéder à l'eau ou bien n'avaient pas la chance de tomber sur un point d'eau. Ce processus a engendré une différenciation de situation entre les attributaires et a favorisé l'émergence de comportements individualistes au détriment de la coopérative.

En 2006, le gouvernement marocain choisit de «libéraliser » le secteur, jugeant d'une part, que les coopératives ne fonctionnaient pas et d'autre part, que la pluralité des statuts fonciers au Maroc rendait la valorisation des terres agricoles difficiles ${ }^{37}$ et enfin, que le modèle de la réforme agraire était figé ce qui entravait l'intensification et l'investissement sur ces terres. Du côté des adhérents, au vu de leur endettement, certains choisirent de vendre leur terre alors que d'autres devinrent propriétaires de la terre sur laquelle ils travaillaient ${ }^{38}$. Ainsi, le secteur de la réforme agraire est en train de se décomposer alors que la valeur de la terre est de plus en plus déterminée par le prix du marché qui a considérablement augmenté depuis 2006, passant d'environ 120000 dirhams ( \pm 11 ooo euros) l'hectare à plus de 500 ooo dirhams ( \pm 45 ooo euros) en 2013 à condition que le terrain soit bien placé (proche d'une ville ou d'une route goudronnée et ait un accès à l'eau). Comme le rapportent certaines personnes interviewées, «seules des personnes riches peuvent se permettre d'engager de telles sommes pour obtenir de la terre ${ }^{39}$. Ou bien, comme l'indiquait une ancienne adhérente : « les personnes qui peuvent acheter de la terre n'ont pas gagné l'argent en travaillant $»^{40}$, évoquant des transactions utilisées pour blanchir de

\footnotetext{
$35 \quad$ Mahdi et Allali 2001.

36 Certains adhérents n'avaient pas suffisamment de moyens pour pouvoir exploiter leur lot de terre. Plusieurs sont ceux qui ont pris des crédits pour ainsi pouvoir faire des investissements sur leur lot de terre.

37 Jouve 2002 ; Akesbi et al. 2007.

38 Ainsi, ils devenaient propriétaire après s'être acquittés du remboursement de la totalité de leur lot et après avoir remboursé leurs dettes.

39 Entretien avec un ancien adhérent de la coopérative effectué le 10 octobre 2013 en darija par l'auteur avec l'aide d'une assistante de recherche/interprète marocaine dans la coopérative d'Ait Ali.

40 Entretien avec une ancienne adhérente effectué le 11 novembre 2013 en darija par l'auteur avec l'aide d'une assistante de recherche/interprète marocaine dans la coopérative d'Ait Ali.
} 
l'argent généré par le cannabis, généralement effectuées par des personnes venues de la région du Rif, au nord du Maroc.

Parallèlement, le secteur agricole bénéficie d'une exonération temporaire d'impôts depuis 1984 (renouvelée en 2013): exonération totale pour les petites et moyennes exploitations et pour les exploitants agricoles réalisant un chiffre d'affaires inférieur à cinq millions de dirhams. Cette situation est particulierement bénéfique pour des professions libérales venues investir dans le secteur agricole, professions qui ne se confrontent pas réellement aux contrôles fiscaux mais profitent de la niche fiscale dans le domaine agricole ${ }^{41}$.

Ainsi, l'État reste fortement impliqué avec une forte présence pour contrôler les dynamiques autour de la libéralisation du secteur de la réforme agraire, offrant des exemptions d'impôts ainsi que des subventions pour de nouveaux investissements ${ }^{42}$. Cela favorise l'émergence d'un secteur agricole considéré comme «productif» et «compétitif», géré par des «agriculteurs entrepreneurs ». Cela entraîne également une augmentation des projets agricoles à grande échelle nécessitant d'importants investissements, une mécanisation, de nouvelles technologies (goutte-à-goutte et forage) et un choix de cultures à forte valeur ajoutée (arbres fruitiers et raisins de table) mais cela fragilise le patrimoine et met en danger le mode de vie de cette agriculture paysanne que nous analysons dans cet article.

\section{Le devenir de l'agriculture paysanne dans un contexte de libéralisation et de modernisation}

Parmi les trente-six adhérents de la coopérative d'Ait Ali, huit ont vendu leurs terres ${ }^{43}$, dix-huit ont obtenu leur titre foncier et les autres restent encore membres de la coopérative. La majorité des terres était vendue à des gens venant d'autres régions du Maroc, d'où l'appellation barrani ce qui signifie quelqu'un qui vient de l'extérieur («étranger» dans le sens où la personne concernée n'est pas originaire du coin). Ils ne s'installent pas sur place, habitant plutôt en ville ou à létranger. Dans notre cas, six des huit acheteurs ont fait d'importants investissements sur les terres qu'ils ont acquises, ce qui a visiblement changé le paysage. Par exemple, ils démarquent leur nouvelle propriété en la clôturant avec des grillages ou des murs et font des cultures pérennes, comme des raisins de table ou des arbres fruitiers. Leur façon de pratiquer

\footnotetext{
41 Voir aussi Mahdi 2005.

42 Différentes subventions sont offertes dans le cadre du « Plan Maroc Vert».

43 Ces chiffres datent de 2013. Depuis, il est possible qu'ils aient changés.
} 
l'agriculture contraste fortement avec celle des adhérents qui sont restés en possession de leurs terres. Deux des acheteurs continuent dans une agriculture mixte, partiellement irriguée et partiellement en bour. Nos interlocuteurs/ trices les nomment «des projets en suspens »: certains acheteurs attendent d'accumuler suffisamment d'argent afin de financer leur projet d'arboriculture fruitière ou de vigne, d'autres choisissent de spéculer en revendant la terre à un prix plus élevé.

C'est dans ce cadre que nous abordons, dans la section suivante, le devenir des différentes trajectoires de familles paysannes en analysant comment les relations de genre et les identités se transforment et se réarticulent dans le contexte étudié.

\section{$1 \quad$ La famille paysanne qui vend sa terre}

Plusieurs histoires que nous avons recueillies des familles qui ont vendu leurs terres étaient tristes et douloureuses : peu de familles concernées étaient disposées à en parler. Lorsque l'on abordait le sujet, les gens avaient soit beaucoup de difficultés à admettre ouvertement la vente de la terre ou bien ils essayaient de nous cacher cette information, déviant la conversation vers d'autres sujets. Leur regret et leur tristesse d'avoir été séparés de leur terre prouve bien que celle-ci représente beaucoup plus qu'une propriété : c'était le résultat d'années de lutte de leurs parents et grands-parents pour obtenir le droit de posséder cette terre. La possession de la terre témoignait aussi de dures années de travail et de beaucoup d'investissements. Lorsque l'on demandait à nos interlocuteurs dans la coopérative pourquoi tant de gens ne voulaient pas admettre avoir vendu la terre, ils nous répondaient : «Vendre la terre, c'est comme vendre ses parents ou sa mère - cela ne se fait pas! ${ }^{44}$.

Les raisons expliquant la vente de la terre diffèrent d'une situation à l'autre. Deux des vendeurs n'étaient pas d'Ait Ali et n'avaient, de ce fait, pas forcément le même rapport à la terre comme l'indique le témoignage suivant d'un ancien adhérent: «Je viens de vendre la terre. Je ne suis pas d'ici et je préfère avoir de l'argent pour construire une maison dans mon village natal » ${ }^{45}$. D'autres ont vendu parce qu'ils étaient trop endettés ou pour éviter des conflits. Dans d'autres cas, alors que des familles élargies travaillaient pendant des années sur une même terre, les vendeurs refusent que celle-ci ne revienne qu'à un

44 Entretien avec une famille du douar d'Ait Ali effectué le 3 juillet 2013 en darija par l'auteur avec l'aide d'une assistante de recherche/interprète marocaine.

45 Entretien avec un ancien adhérent de la coopérative effectué le 2 novembre 2013 en darija par l'auteur avec l'aide d'une assistante de recherche/interprète marocaine dans la coopérative d'Ait Ali. 
seul héritier; pour éviter des conflits, ils choisissent alors de vendre la terre et de s'en partager le revenu. Dans le meilleur des cas, c'est une solution mais il arrive aussi qu'un seul héritier touche tout l'argent.

Les familles qui ont choisi ou été obligées de vendre leur terre sont souvent mal perçues par les autres familles dans la coopérative : elles sont fréquemment qualifiées comme «gaspilleuses». Quelques familles sont parties en ville et ont acheté des maisons et des voitures ou ont fait le pèlerinage à la Mecque. D’autres, ayant dépensé tout leur argent en ville, ont donc été obligées de revenir dans la coopérative et, n'ayant aucun autre moyen de générer des revenus, leurs membres deviennent ouvriers et finissent par travailler sur les terres qui leur appartenaient au temps jadis: "Les adhérents qui se trouvent dans cette situation perdent leur dignité » ${ }^{46}$. Cependant, leurs fils chercheront à se professionnaliser dans le travail agricole alors que les jeunes ouvrières sont souvent méprisées et stigmatisées dans la société rurale du Saïss ${ }^{47}$.

Cette catégorie de famille a obtenu son titre foncier et cherche à moderniser l'exploitation de la terre. Pour obtenir son titre foncier, la majorité des familles ont vendu leur lot irrigué de 0,5 hectare pour en couvrir les frais. Aujourd'hui, elles commencent à creuser de nouveaux forages pour augmenter la surface irriguée afin de planter plus de légumes et prévoient, ou sont déjà en train, d'installer un système de goutte-à-goutte et de planter des arbres fruitiers sur quelques hectares.

Les jeunes hommes dont le père ou la mère a obtenu le titre foncier se lancent dans la modernisation de l'exploitation familiale et se destinent à succéder à leur père en tant que chef d'exploitation. Les projets agricoles des barrani - même si d'habitude les personnes habitant les coopératives ne sont pas directement en contact avec eux - deviennent souvent une source d'inspiration pour ces jeunes et « l'air moderne » les tente. L'évolution technologique contribue à réduire l'effort physique pour les agriculteurs, tout en augmentant la productivité de leur travail, accompagnée d'une gestion professionnelle de l'exploitation. Cette approche nouvelle offre à ces jeunes agriculteurs la possibilité de se distinguer positivement par rapport à leurs parents paysans qu'ils qualifient de «traditionnels». Ils deviennent de nouveaux agriculteurs «propres» avec un air d'«entrepreneur moderne». L'exemple de Khalid

46 Entretien avec un adhèrent de la coopérative et les membres de sa famille effectué le 14 juin 2013 par l'auteur avec l'aide d'une assistante de recherche/interprète marocaine dans la coopérative d'Ait Ali. 
montre bien comment les jeunes hommes sont interpellés par les nouvelles possibilités qui deviennent réalisables du fait que le père ou la mère possède la terre. Dans le cas de Khalid (âgé de 26 ans), son père était adhérent et la terre venait d'être mise à son nom ${ }^{48}$. Auparavant, Khalid travaillait dans des fermes dans la région d'Agadir. Il est revenu au bled pour travailler avec son frère sur la terre familiale : «Avant, je partais travailler à Agadir dans les serres. Mais maintenant je ne pars plus et je travaille ici. Avec mon frère, on est en train de penser à faire quelque chose pour nous même et de ne plus travailler pour le compte de quelqu'un d'autre. On vient d'installer le goutte-à-goutte et on pense créer des étables ».

Pour ces familles, la possession de la terre joue le rôle d'un ascenseur social et leur statut a progressé : d'ouvrier à attributaire, à un statut de propriétaire d'une terre qui vaut aujourd'hui environ 500 ooo dirhams l'hectare. Du fait de leur nouveau statut et de la pratique de nouvelles cultures demandant plus de main d'œuvre, les familles embauchent de plus en plus d'ouvriers en dehors de la famille et même de la communauté. La présence d'ouvriers venant de l'extérieur, surtout quand il s'agit d'hommes, rend les femmes plus réticentes à travailler sur l'exploitation comme en témoigne Khadija (âgée de 30 ans) : «On avait l'habitude de travailler sur la terre lorsque cétait nécessaire. Par exemple, pendant la récolte des oignons, je remplissais en général les caisses. Aujourd'hui, je le fais rarement car la récolte est faite par des ouvriers (hommes). On ne travaille pas ensemble avec eux $\gg^{49}$.

Alors que l'épouse de l'adhèrent initial vieillit, les femmes des héritiers plus jeunes consacrent leur temps au travail domestique ou s'occupent des étables ou des activités agricoles proches de la maison : «Je ne travaille plus que rarement sur la terre. Mais je cuisine quand les ouvriers viennent, je m'occupe de la maison et des enfants. Ce que je fais est le moteur de l'exploitation!». Tout en étant confinées dans la sphère domestique, les femmes de cette génération revendiquent l'importance de leur travail, affirmant ainsi leur évolution sociale. En revanche, les jeunes femmes (filles de parents paysans) ne se contentent pas uniquement de ce nouveau rôle, elles cherchent leur indépendance et sont à la poursuite d'un autre modèle féminin. Elles veulent professionnaliser leurs

48 Entretiens avec Khalid effectués le 10 juin, 24 juin et 12 novembre 2013, en darija par l'auteur avec l'aide d'une assistante de recherche/interprète marocaine dans la coopérative d'Ait Ali.

49 Entretien avec l'épouse d'un adhérent de la coopérative effectué le 27 août 2013 en darija par l'auteur avec l'aide d'une assistante de recherche/interprète marocaine dans la coopérative d'Ait Ali. 
activités domestiques : créer une pâtisserie dans le village, un projet laitier, monter un atelier de couture ou vendre des vêtements aux voisines ${ }^{50}$.

\section{$3 \quad$ La famille paysanne qui se maintient}

Il ne reste dans la coopérative d'Ait Ali que dix familles qui n'ont pas encore obtenu leur titre foncier, elles vivotent et se trouvent dans une impasse. Soit elles n'ont pas les moyens de rembourser leurs dettes, soit elles se trouvent empêtrées dans des conflits entre héritiers et attendent que le titre sorte pour pouvoir diviser la terre. En effet, depuis le processus de «melkisation », le système d'héritage particulier qui existait dans les coopératives a été remplacé par les règles de la shari'a, sur laquelle la législation marocaine est fondée, qui fixe, pour les femmes, une part d'héritage inférieure à celles des hommes ${ }^{51}$. La famille de Mohammed (âgé de 21 ans), qui a deux oncles et deux tantes, se trouve dans une telle situation de maintien ${ }^{52}$. Le grand-père de Mohammed, décédé en 2013, était adhérent de la coopérative. La demande du titre foncier avait était lancée juste avant son décès. En principe, la demande devait être réalisée dans un délai de six mois mais, en pratique, cela peut durer plus de six mois, voire des années. Les deux oncles de Mohammed veulent continuer à travailler la terre collectivement mais les sœurs et le père de Mohammed veulent la partager ; les sœurs pour vendre leurs parts et le père, qui ne s'entend pas avec ses frères, veut la garder pour y travailler seul. Ainsi, tandis que Mohammed voit changer le paysage autour de lui, sa famille continue de cultiver, sur les quelques hectares irrigués, des oignons ou des pommes de terre et, sur le reste du lot, des céréales et des cultures fourragères. Les oncles de Mohammed font rarement appel à des ouvriers de l'extérieur. Toute la famille reste impliquée dans le travail agricole comme pendant la période de la coopérative: les frères et leur femme ainsi que les filles non-mariées travaillent ensemble sur la terre, les différentes tâches étant bien réparties selon le genre comme auparavant.

Avec le changement du statut de la terre et du domaine de l'agriculture qui devient plus professionnel, les femmes ne trouvent plus beaucoup de reconnaissance dans leur travail agricole. Elles voient comment leurs voisins qui ont obtenu leur titre foncier modernisent leur exploitation et embauchent une

\footnotetext{
50 Bossenbroek, Van der Ploeg et Zwarteveen 2015.

51 Daoudi 2012.

$5^{2}$ Entretiens avec Mohammed et les membres de sa famille effectués le 26 juin 2012, 29 juin et 31 octobre 2013, par l'auteur avec l'aide d'une assistante de recherche/interprète marocaine dans la coopérative d'Ait Ali.
} 
main d'œuvre extérieure alors qu'elles sont fatiguées du travail sur la terre ${ }^{53}$ : «Ce n'est pas une vie, on travaille du matin au soir». En particulier, les jeunes femmes cherchent à échapper à la «corvée » comme le montre l'exemple suivant de Halima (âgée de 23 ans) : «J'aide mon papa sur la terre. J'aide pendant la période de l'oignon, on fait la plantation et on fait nous-même le désherbage et on récolte les oignons qu'on installe sur la terre. Mes frères, mon papa et mes oncles les récoltent de la terre. Je ne fais rien d'autre. J'aimerais bien me marier avec quelqu'un en ville et partir habiter là-bas ». Avec peu de divertissements et d'autres options que le travail agricole et en voyant le changement autour d'eux sans le sentir dans leur propre vie, certaines jeunes femmes comme Halima veulent partir en ville pour ne pas suivre le destin de leur mère. D'autres cherchent à se libérer un peu de leur corvée et à participer aux cours d'alphabétisation ou de Coran qui sont mis en place dans le douar.

Les jeunes hommes de ces familles observent comment leurs parents stagnent comparés aux barranis et aux familles qui ont obtenu leur titre foncier. Alors que le monde autour d'eux change, comme les jeunes femmes, ils sentent que cela a peu d'effet sur leur situation personnelle et ne trouvent pas de possibilité pour moderniser ou transformer leur exploitation. Inspirés par les changements dans le domaine de l'agriculture, ils ne veulent pas reproduire les mêmes identités traditionnelles que leurs parents. Ils préfèrent alors travailler ailleurs sur d'autres exploitations ou partir en ville dans l'espoir de revenir un jour pour prendre la relève selon leurs conditions et souhaits.

\section{Conclusion}

À travers notre analyse des trajectoires de différentes familles paysannes et des dynamiques actuelles autour de la libéralisation des terres des coopératives de la réforme agraire, nous avons analysé comment l'agriculture familiale de ce domaine est en mutation. Pendant la période de la coopérative, la terre, l'exploitation et la famille paysanne étaient intimement liées. Les activités et l'organisation autour de la terre forment les identités des hommes et des femmes paysans. Ceci contribua à une démarcation floue entre famille paysanne et exploitation, la terre étant presque une extension logique du domaine domestique. Or, aujourd'hui, la libéralisation des terres des coopératives de la

53 Entretiens avec une jeune femme âgée de 28 ans dans la coopérative effectué le 26 juillet 2013 par l'auteur avec l'aide d'une assistante de recherche/interprète marocaine dans la coopérative d'Ait Ali. 
réforme agraire modifie ces relations. Ceci est dû aux changements attribués à la valeur de la terre. Pour les familles paysannes qui ont obtenu leur titre foncier, la terre reflète leur évolution sociale. Elles ont vaincu l'histoire et ont les moyens d'embaucher des ouvriers. Dans ce contexte, les femmes paysannes, tout en étant confinées dans la sphère domestique, revendiquent l'importance de leur travail dans et autour de la maison, affirmant ainsi leur nouveau statut social. Elles ne cherchent plus à travailler sur la terre. D'autant plus, que la croissance de nouveaux projets agricoles induit une certaine professionnalisation masculine du domaine de l'agriculture et la femme n'éprouve plus de fierté dans le travail agricole.

Pour les jeunes hommes dont les pères ou les mères étaient adhérents et qui ont obtenu leur titre foncier, la professionnalisation des activités et du secteur agricole offre de nouvelles opportunités attirantes qui leur permettent de se distinguer de leurs parents «paysans » et de devenir un nouveau type d'agriculteur plus moderne. Par contre, les jeunes femmes ne voulant pas reproduire les mêmes identités féminines que leur mère cherchent à se professionnaliser selon leurs conditions mais sans avoir de modèle ou d'exemple à suivre.

Notre analyse montre que ces dynamiques stigmatisent les familles qui ont vendu et qui sont devenues ouvrières et, en particulier, la femme ouvrière. Alors que ces familles observent l'ascension sociale de leurs voisins, elles sont devenues des paysans sans terre, des prolétaires.

Finalement, sous l'angle de ces changements, on s'aperçoit que dans les familles paysannes se trouvant dans une impasse, sans projection dans l'avenir, la femme a peu de fierté quant à ses activités menées sur la terre dues à la professionnalisation et masculinisation du domaine agricole. Les jeunes femmes cherchent à vivre dans de meilleures conditions souvent en ville alors que les jeunes hommes ont la possibilité d'aller travailler ailleurs dans l'agriculture ou un autre domaine, tout en se voyant revenir un jour prendre la relève de l'exploitation.

Ainsi, plus de quarante ans après la mise en place des coopératives de la réforme agraire, le secteur est en train d'être complètement « détricoté » et les familles sont laissées à leur sort et au pouvoir du marché. Souvent en marge des politiques publiques et non prises en compte, c'est justement la jeunesse rurale qui forme le maillon du devenir de cette agriculture familiale. Alors que la nouvelle génération d'agriculteurs projette à court ou à long terme de prendre la relève de l'exploitation, tout en l'adoptant à leurs souhaits, les jeunes femmes sont à la recherche d'un nouveau modèle féminin qui leur permettrait de se professionnaliser et d'élargir leurs travaux domestiques ou de trouver de nouvelles niches. En trouvant peu de place dans les dynamiques 
actuelles, certaines cherchent à partir en ville, ce qui aura des répercussions sur cette agriculture familiale.

\section{Bio}

Lisa Bossenbroek obtained her PhD in rural sociology at the University of Wageningen (the Netherlands). As part of her research she studied the role of young people in agrarian dynamics and the interactions of processes of agrarian change and gender relations. Currently, she works as a post-doc at the Faculty of Governance, Economics and Social Sciences (EGE-RABAT), Morocco. She has worked extensively in Central Asia and North Africa. Currently she is conducting comparative research that focuses on the interaction of agrarian change and gender relations and subjectivities in Morocco and India.

\section{Références}

\section{Sources de première main}

Pour le corpus d'entretiens menés pour ce travail, voir Lisa Bossenbroek, 2016, Behind the veil of agricultural modernization: gendered dynamics of rural change in the Saiss, Morocco, Wageningen, Wageningen University, The Netherlands, p. 52

\section{Publications}

Akesbi, Najib, Driss Benatya et Noureddine El Aoufi, 2007, «Les implications structurelles de la libéralisation sur l'agriculture et le développement au Maroc », Colloque International: Enjeux économiques, sociaux et environnementaux de la libéralisation commerciale des pays du Maghreb et du Proche-Orient, Rabat: https://www.gate .cnrs.fr/uneca07/communications\%2opdf/El\%20Aoufi\%20-\%2oRabat.pdf.

Akesbi, Najib, 2012, «Une nouvelle stratégie pour l'agriculture marocaine: le 'Plan Maroc Vert' », New Medit. A Mediterranean Journal of Economics, Agriculture and Environment 11, pp. 12-23.

Anglade, Marie-Pierre, Leïla Bouasria, Mériem Cheikh, Fanny Debarre, Véronique Manry et Camille Schmoll, 2013, Expériences du genre. Intimités, marginalités, travail et migration, Paris, Karthala / Casablanca, Éditions Le Fennec.

Argent, Neil, 1999, "Inside the Black Box: Dimensions of Gender, Generation and Scale in the Rural Restructuring Process", Journal of Rural Studies 15, pp. 1-15.

Asztalos, Morell Ildikó and Bertit Brandth, 2007, "Family and Gender in the Transformation of the Countryside", Journal of Comparative Family Studies 38, pp. 371-77. 
Barthez, Alice, 2005, « Devenir agricultrice : à la frontière de la vie domestique et de la profession », Économie Rural, 289-29o, pp. 30-43.

Belarbi, Aïcha, 1995, Femmes Rurales, Casablanca, Éditions le Fennec.

Bossenbroek, Lisa, 2016, Behind the veil of agricultural modernization: gendered dynamics of rural change in the Saiss, Morocco, Wageningen, Wageningen University, The Netherlands.

Bossenbroek, Lisa, Jan Douwe Van der Ploeg and Margreet Zwarteveen, 2015, "Broken Dreams? Youth Experiences of Agrarian Change in Morocco's Saïss Region”, Cahiers Agricultures 24, pp. 342-48.

Bossenbroek, Lisa and Margreet Zwarteveen, 2015, “One Doesn't Sell One's Parents: Gendered Experiences of Land Privatization in the Sais, Morocco", 2015 in Caroline Archambault and Annelies Zoomers, ed., Global Trends in Land Tenure Reform: Gender Impacts, London / New York: Routledge, pp. 152-69.

Bossenbroek, Lisa, Mostafa Errahj et Najoua El Alime, 2016, « es nouvelles modalités du travail agricole dans le Sais au Maroc. L'émergence des inégalités identitaires entre l'ouvrier et l'ouvrière ?» in Baudouin Dupret, Zakaria Rhani, Assia Boutaleb et Jean-Noël Ferrié, éd., Le Maroc au Present, Fondation Abdulaziz / Centre Jacques Berque, Casablanca.

Bouami, Abdelaziz, 1980, « L'Etat et la question agraire », in Parti du Progrès et du Socialisme, La question agraire au Maroc, Casablanca, Al Bayane, pp. 87-129.

Bouasria, Leila, 2013, Les ouvrières marocaines et mouvement, Paris, L'Harmattan.

Bouasria, Leila et Mériem Cheikh, 2013, «Les aléas du genre : conflits, négociations, recompositions » in Marie-Pierre Anglade et al., dir., Expériences du genre. Intimités, marginalités, travail et migration, Paris, Karthala / Casablanca, Éditions Le Fennec.

Bouderbala, Négib, 2001, « La lutte contre le morcellement au Maroc : un thème idéologique » in Anne-Marie Jouve, éd., Terres méditerranéennes. Le morcellement, richesse ou danger?, Paris, Karthala-Ciheam, pp. 41-57.

Bouderbala, Négib et Rachid Filali-Meknassi, 1991, Code Agraire Marocain, Kénitra, Office du Gharb.

Brandth, Berith, 2002, "Gender Identity in European family farming: A Literature Review", Sociologica Ruralis 42, pp. 181-200.

Daoudi, Fatiha, 2001 (octobre), «Droits fonciers des femmes au Maroc: entre complexité du systeme foncier et discrimination », Les Etudes et Essais du Centre Jacques Berque, ${ }^{\circ}{ }_{4}$, Rabat, pp. ${ }^{-1} 35$.

Jouve, Anne-Marie, 2002, «Cinquante ans d'agriculture marocaine» in P. Blanc, éd., Du Maghreb au Proche Orient : les défis de l'agriculture, Paris, L'Harmattan, pp. 51-71. Mahdi, Mohamed et Khalil Allali, 2001, « Les coopératives de la réforme agraires: trente ans après », Publication du bulletin économique et social du Maroc, Rabat, Maroc, Société d'Etudes Economiques Sociales et Statistiques, pp. 109-25.

Mahdi, Mohamed, 2005, Agriculteurs citadins, Meknès, Université Moulay Ismail. 
Mernissi, Fatima, 1982 (fall), "Women and the Impact of Capitalist Development in Morocco, Part I", Feminist Issues, pp. 69-104.

Mernissi, Fatima, 1983 (spring), "Women and the Impact of Capitalist Development in Morocco, Part II", Feminist Issues, pp. 61-112.

Ministère de l'Agriculture et de la Pêche Maritime, 2012, Situation de l'agriculture marocaine, Rabat.

Ministère de l'Agriculture, du Développement Rural et des Pêches Maritimes, 2005, Situation de l'agriculture marocaine, dossier : le foncier agricole, Rabat.

Ministère de l'Agriculture et de la Réforme Agraire, 1973 (26 septembre), «Réforme Agraire ; attribution à des agriculteurs de terres agricoles ou à vocation agricole faisant partie du domaine privé de l'Etat », Bulletin Officiel $\mathrm{n}^{\circ} 3178$.

Ministère de l'Intérieure, 1964, «Circulaire du Ministère de l'Intérieur en date du 27 décembre 1964, relative à un projet de publication d'un recueil de circonscription administrative » in Mohamed Rfass, 1996, L'organisation urbaine de la péninsule Tingitane, Rabat, Université Mohamed v, Publications de la Faculté des Lettres et des Sciences Humaines.

Ní Laoire, Catriona, 2002, "Masculinities and Change in Rural Ireland", Irish Geography 35, pp. 16-28.

Pascon, Paul, 1977, «Interrogations autour de la réforme agraire » in Négib Bouderbala, Mohemd Chraïbi et Paul Pascon, éd. La question agraire au Maroc 2, Publication du Bulletin économique et social du Maroc, pp. 183-200.

Rassam, Amal, 1980, "Women and Domestic Power in Morocco", International Journal of Middle Eastern Studies 12 (2), pp. 171-79.

Whatmore, Sarah, 1991, "Life Cycle or Patriarchy? Gender Divisions in Family Farming", Journal of Rural Studies 7 (1-2), pp. 71-6.

Zwarteveen, Margreet and Marina Endeveld, 1995, "Rural Women's Questions are Agrarian Questions. A Discussion of the Intellectual and Political Construction of Realities of Rural Women", Paper presented at the conference: Agrarian Questions: The Politics of Farming, 1995, Wageningen, Université de Wageningen, Pays-Bas. 